Institut für Theoretische Physik der Universität Regensburg

April 1994

TPR-94-7

\title{
Nucleon Structure Functions from Relativistic Constituent Quarks ${ }^{\text {* }}$
}

\author{
W.Melnitchouk and W.Weise \\ Institut für Theoretische Physik \\ Universität Regensburg \\ D-93040 Regensburg, Germany
}

\begin{abstract}
We investigate deep inelastic lepton scattering from the nucleon within a constituent quark picture, in which the internal structure of constituent quarks is modeled by meson and diquark dressing. In a covariant framework this structure leads to a breakdown of the factorization necessary for a convolution formulation. We perform our analysis in time-ordered perturbation theory in the infinite momentum frame where factorization of subprocesses is automatic. Numerical results are compared with recent data on valence quark distributions in the proton.
\end{abstract}

PACS numbers: 12.40.Aa, 13.60.Hb, 25.30.Fj

${ }^{*}$ Work supported by BMFT grant 06 OR 735.

To appear in Physics Letters B. 
Many attempts have been made to link the parton picture of the nucleon, which is evident in high-energy processes such as deep inelastic scattering (DIS), to the familiar quark models of hadrons which successfully describe much of the low-energy phenomenology. Most calculations of nucleon quark distributions thus far can be grouped into two broad categories - those which use various bag or soliton models of the nucleon [1], and those based on the non-relativistic quark model [2]. More recently, an alternative formulation in terms of relativistic quark-nucleon vertex functions [3] has led to some encouraging results. The advantage here is that one deals directly with covariant Feynman diagrams; on the other hand, without decomposing the vertex functions into explicit nucleon-quark and nucleon-antiquark components the connection between the vertex functions and quark and antiquark probability distributions in a general reference frame is not obvious.

In this paper we suggest how one may combine the advantages of some of the existing approaches - maintaining a close link with the constituent quark picture of the nucleon while at the same time using a formalism which is fully relativistic. We adopt the traditional view that constituent quarks (CQs) themselves have a non-trivial internal structure, which can in principle be resolved by a high-energy probe. In the impulse approximation, DIS from the nucleon can then be viewed as a two-step process, in terms of virtual photon-CQ and CQ-nucleon subprocesses.

The framework which we use is based on time-ordered perturbation theory in the infinite momentum frame (IMF) [4. Since here only on-mass-shell particles are involved, factorization of subprocesses is automatic. Therefore one may express the momentum distribution of quarks inside a nucleon $\left(q_{N}\right)$ as a convolution of a constituent quark momentum distribution in a nucleon $\left(f_{Q / N}\right)$ and the structure function of a constituent quark $\left(q_{Q}\right)$. Factorization is generally not possible in a covariant framework where particles are off-mass-shell. In any frame other than the IMF one must include in addition diagrams with particles moving backwards in time ("Z-graphs"), which will introduce corrections to any convolution formula.

To describe DIS from a CQ we consider a simple dynamical model, namely the Nambu \& Jona-Lasinio (NJL) model [5, 6], in which gluonic degrees of freedom are integrated out and absorbed into an effective point-like quark interaction, characterized by an effective coupling constant, $g$. Since the NJL model is constructed to approximate QCD in the low-energy and long-wavelength limit, the CQ distributions 
will be those at a scale relevant to the model itself $\left(Q^{2}=Q_{0}^{2} \lesssim 1 \mathrm{GeV}^{2}\right)$. Explicit perturbative gluonic degrees of freedom reappear when the calculated distributions are evolved to the higher $Q^{2}$ appropriate for the DIS region. This procedure may be questionable since one assumes the validity of perturbative QCD in a region where higher order corrections are not negligible. On the other hand, the next-to-leading order analysis of Ref.[7] (which uses $\left.Q_{0}^{2} \sim(0.4 \mathrm{GeV})^{2}\right)$ indicates 10-20\% effects at intermediate $x$ when two-loop radiative corrections are included.

In the NJL model the CQ structure function is determined by the forward quarkquark $(Q-Q)$ scattering amplitude [8], which at zeroth-order in $g$ gives rise to the process depicted in Fig.1(a). In this case the CQ structure function is a deltafunction, and the resulting nucleon quark distribution is given by the function $f_{Q / N}^{(S)}$ :

$$
q_{N}^{\operatorname{bare}(S)}(x)=\int_{x}^{1} \frac{d y}{y} \int_{0}^{\infty} d p_{T}^{2} f_{Q / N}^{(S)}\left(y, p_{T}\right) \delta\left(1-x_{Q}\right)=\int_{0}^{\infty} d p_{T}^{2} f_{Q / N}^{(S)}\left(x, p_{T}\right)
$$

where $x=Q^{2} / 2 P \cdot q$ is the Bjorken scaling variable, $y=p \cdot q / P \cdot q$ is the fraction of the nucleon's momentum carried by the constituent quark, and $x_{Q}=x / y$ is the fraction of the constituent quark's momentum carried by the struck quark. In Eq.(1) the superscript $S$ denotes a quark distribution associated with a spectator diquark system in a spin $S=0$ or $S=1$ state. The label "bare" refers to a constituent quark that is not dressed by $Q-Q$ interactions.

Beyond the trivial order in $g$, in the pole approximation the $t$-channel amplitude is well approximated (at least in the pseudoscalar sector) by using meson propagators, and by diquark propagators in the $s$-channel. This naturally gives rise to a dressing of the constituent quark by meson and diquark "clouds", Figs.1(b) and (c), which has the effect of softening the hard, $\delta$-function, "bare" quark distribution in Eq.(1). The $s$-channel diquark-exchange process in Fig.1(c) in fact generates an antiquark component of the constituent quark, which in turn provides a simple mechanism by which a non-perturbative sea can arise in the nucleon. The dressing corrections will be most relevant in the region of small $x$, and higher order corrections involving additional ladders of exchanged mesons and diquarks will not be relevant for valence quark distributions, on which we focus in the present work.

Having calculated their structure within a model, we assume that CQs embedded in a nucleon have some characteristic momentum distribution, which in the present approach is determined by the relativistic quark-diquark-nucleon $(Q D N)$ 
vertex function, $\Phi_{Q D N}^{(S)}$. The Dirac structure and momentum dependence of the $Q D N$ vertex can in principle be very complicated, and our treatment at this point is phenomenological. Following earlier work [3, 9] we approximate the vertex function by two structures, one for scalar and one for pseudovector diquarks. A more elaborate treatment of vertex functions, for example by solving covariant Faddeev or Bethe-Salpeter equations [10], is beyond the scope of the present work, but will be dealt with elsewhere [14].

The CQ structure function and the $Q D N$ vertex function then determine the valence quark distribution in the nucleon:

$$
q_{N}^{\operatorname{val}(S)}(x)=Z q_{N}^{\operatorname{bare}(S)}(x)+\delta^{(M)} q_{N}^{(S)}(x)-\delta^{(D)} \bar{q}_{N}^{(S)}(x)
$$

where the three terms correspond to the "bare", meson-exchange, and diquarkexchange processes in Figs.1(a), (b) and (c), respectively. At order $g^{2}$ one could also include the process where a diquark is struck, which would require modeling in addition the structure function of an off-shell diquark [11]. In the present analysis we do not consider these processes explicitly, but rather assume that they can be absorbed into the effective $Q D N$ vertex in Fig.1(a) (see below). The consequences for the valence quark distributions will be negligible provided the normalization constant $Z$ is chosen to ensure overall baryon number conservation. Direct scattering from a pion, however, would enter only at $\mathcal{O}\left(g^{4}\right)$.

For a scalar $(S=0)$ diquark we consider a vertex $\propto\left(\not p-\not p^{\prime}\right) / M$, which gives:

$$
\begin{aligned}
f_{Q / N}^{(0)}\left(x, p_{T}\right) & =\frac{1}{16 \pi^{2}} \frac{\left|\Phi_{Q D N}^{(0)}\left(x, p_{T}\right)\right|^{2}}{x(1-x)\left(M^{2}-s_{Q D}\right)^{2}} \frac{1}{M^{2}}\left(2 M m_{Q}\left(m_{D}^{2}+m_{Q}^{2}\right)\right. \\
+2 & \left.P \cdot p\left(m_{Q}^{2}-m_{D}^{2}\right)-4 M m_{Q} p \cdot p^{\prime}-4 m_{Q}^{2} P \cdot p^{\prime}+4 P \cdot p^{\prime} p \cdot p^{\prime}\right)
\end{aligned}
$$

where $m_{Q(D)} \simeq 0.4(0.6) \mathrm{GeV}$ is the constituent quark (scalar diquark) mass, and $s_{Q D} \equiv\left(p+p^{\prime}\right)^{2}=\left(m_{Q}^{2}+p_{T}^{2}\right) / x+\left(m_{D}^{2}+p_{T}^{2}\right) /(1-x)$ is the squared mass of the $Q-D$ system. To generate sufficiently hard quark distributions with a structure $\propto I$ [3, 9, 11] one would need more singular behavior in $p_{T}$ of the vertex function $\Phi_{Q D N}^{(0)}$ than the simple dipole form used below.

Any realistic model of the nucleon must also incorporate pseudovector diquarks. We model the quark distribution for an $S=1$ diquark with a vertex $\gamma_{5} \gamma_{\alpha}$, which 
gives:

$$
f_{Q / N}^{(1)}\left(x, p_{T}\right)=\frac{1}{16 \pi^{2}} \frac{\left|\Phi_{Q D N}^{(1)}\left(x, p_{T}\right)\right|^{2}}{x(1-x)\left(M^{2}-s_{Q D}\right)^{2}}\left(6 M m_{Q}+2 P \cdot p+\frac{4 P \cdot p^{\prime} p \cdot p^{\prime}}{m_{D}^{2}}\right) .
$$

The different $S=0$ and $S=1$ vertices, as well as a larger pseudovector diquark mass $\left(m_{D} \simeq 0.8 \mathrm{GeV}\right)$, explicitly break spin-flavor SU(4) symmetry. This not only provides a simple explanation of the $\Delta-N$ mass difference, but also the large- $x$ $F_{2 n} / F_{2 p}$ ratio 12.

Contributions to nucleon quark distributions from DIS off dressed CQs quarks can also be formulated as convolutions, similar to Eq.(11). This is only possible, however, because of the on-mass-shell condition for interacting particles in the timeordered theory. In a covariant formalism the non-trivial Dirac structure of the $\gamma^{*}-Q$ scattering amplitude and the off-shell dependence of the $Q D N$ vertex render factorization invalid. This can be demonstrated by writing the CQ structure function as:

$$
q_{Q}\left(x_{Q}\right)=\operatorname{Tr}\left[\left(\not p+m_{Q}\right) \widehat{q}_{Q}\left(x_{Q}, p^{2}\right)\right]
$$

where the operator $\widehat{q}_{Q}=I \widehat{q}_{0}+\not p \widehat{q}_{1}+\not q \widehat{q}_{2}$ describes DIS from an off-shell CQ. In terms of the coefficients $\widehat{q}_{0 \cdots 2}$ the CQ structure function can then be written:

$$
q_{Q}\left(x_{Q}\right)=4 m_{Q} \widehat{q}_{0}+4 m_{Q}^{2} \widehat{q}_{1}+4 p \cdot q \widehat{q}_{2} .
$$

For a point-like quark, in the scaling limit one has $\widehat{q}_{2} \neq 0$, while $\widehat{q}_{0,1}=0$. On the other hand, for a CQ with internal structure all three coefficients can be non-zero. Indeed, dressing by either a meson or diquark cloud gives $\widehat{q}_{0}=\mathcal{O}(1), \widehat{q}_{1}=\mathcal{O}(1)$, $\widehat{q}_{2}=\mathcal{O}\left(1 / q^{2}\right)$. In terms of $\widehat{q}_{Q}$ the quark distribution in the nucleon is:

$$
q_{N}(x)=\int d^{4} p \operatorname{Tr}\left[\left(I \mathcal{A}_{0}\left(p^{2}\right)+\gamma_{\alpha} \mathcal{A}_{1}^{\alpha}\left(p^{2}\right)\right) \widehat{q}_{Q}\left(x_{Q}, p^{2}\right)\right]
$$

where the functions $\mathcal{A}_{0,1}$ represent the off-shell $Q-N$ interaction. Because of the scaling properties of $\widehat{q}_{0 \cdots 2}$, the trace in Eq.(7) will not be proportional to the CQ structure function $q_{Q}$ in Eq.(河). Therefore Eq.([) can only be written in convolution form if the functions $\mathcal{A}_{0,1}$ are proportional, however this will not be the case either unless the on-mass-shell limit is taken for these. This result is analogous to that for an off-shell nucleon bound inside a nucleus [9]. However, unlike in the nuclear case where nucleon off-shell corrections are not overwhelming [9, 13, neglect of quark 
off-shell effects in the nucleon can lead to significant errors. On the other hand, because of the on-mass-shell kinematics in the time-ordered approach, the $Q D N$ interaction factorizes, allowing $\delta^{(M)} q_{N}^{(S)}$ and $\delta^{(D)} \bar{q}_{N}^{(S)}$ in Eq.(2) to be expressed as two-dimensional convolutions as in Eq.(1).

For the meson dressing process in Fig.1(b) we restrict ourselves to pions. Dressing of constituent quarks by higher mass mesons (e.g. $\rho, \omega$, etc.) can be considered, but contributions from these will be suppressed due to the larger meson masses. The contribution to $q_{N}$ from a constituent quark dressed by a pion is given by:

$$
\delta^{(\pi)} q_{N}^{(S)}(x)=\int_{x}^{1} \frac{d y}{y} \int_{0}^{\infty} d p_{T}^{2} f_{Q / N}^{(S)}\left(y, p_{T}\right) \int d^{2} k_{T} q_{Q}\left(x_{Q}, k_{T}, p_{T}\right)
$$

where $f_{Q / N}^{(S)}$ is given by Eqs.(3) and (4), and

$$
\begin{aligned}
q_{Q}\left(x_{Q}, k_{T}, p_{T}\right)= & \frac{g_{Q \pi}^{2}}{16 \pi^{3}} \frac{\left|\Phi_{Q Q \pi}\left(x_{Q}, k_{T}, p_{T}\right)\right|^{2}}{x_{Q}\left(1-x_{Q}\right)\left(m_{Q}^{2}-s_{Q \pi}\right)^{2}} \\
& \times\left(\frac{k_{T}^{2}+m_{Q}^{2}\left(1-x_{Q}\right)^{2}}{x_{Q}}+x_{Q} p_{T}^{2}-2 \mathbf{p}_{T} \cdot \mathbf{k}_{T}\right)
\end{aligned}
$$

represents the $\gamma^{*}-\left(\right.$ dressed) CQ interaction. Here $g_{Q \pi}=m_{Q} / f_{\pi} \approx 4$ is the $Q_{-}$ $\pi$ coupling constant [6], as given by the Goldberger-Treiman relation, and $s_{Q \pi} \equiv$ $\left(k+k^{\prime}\right)^{2}=\left(m_{Q}^{2}+k_{T}^{2}\right) / x_{Q}+\left(m_{\pi}^{2}+\left(\mathbf{p}_{T}-\mathbf{k}_{T}\right)^{2}\right) /\left(1-x_{Q}\right)-p_{T}^{2}$. In terms of $q_{Q}\left(x_{Q}, k_{T}, p_{T}\right)$ the structure function of a (free) constituent quark at rest (Eq.(5)) is: $q_{Q}\left(x_{Q}\right)=\int_{0}^{\infty} d k_{T}^{2} q_{Q}\left(x_{Q}, k_{T}, 0\right)$.

The presence of transverse momentum $p_{T}$ in both $f_{Q / N}$ and $q_{Q}$ in Eq.(8) means that a simple one-dimensional convolution in $y$ alone cannot be valid unless the $p_{T}$-dependence in $q_{Q}$ is neglected. This turns out to be a rather bad approximation, however, as the $p_{T}=0$ results are found to differ by up to $30-40 \%$ from the full, $p_{T}$-dependent calculation of the pion-dressed contributions [14].

In Eq.(8) we assume that the integral of $q_{Q}$ over $k_{T}$ is regularized by a sharp cutoff $\Lambda$, and consequently parametrize the $Q Q \pi$ vertex function by a theta-function, $\Phi_{Q Q \pi}=\theta\left(k_{T}^{2}-\left(1-x_{Q}\right) \Lambda^{2}\right)$, with $\Lambda \approx 0.9 \mathrm{GeV}$ [6] characteristic of a typical chiral symmetry breaking scale. The contribution of the diquark-exchange process in Fig.1(c) to the nucleon antiquark distribution is similar to the $\pi$ contribution in Eq.(8),$\quad \delta^{(\pi)} q_{N}^{(S)}(x) \rightarrow \delta^{(D)} \bar{q}_{N}^{(S)}(x)$, but with $m_{\pi} \rightarrow m_{D}$ and $g_{Q \pi} \rightarrow g_{Q D} \approx 4$ taken from the NJL model.

The only additional input necessary for the numerical evaluation of $q_{N}^{\operatorname{val}(S)}(x)$ is the $Q D N$ vertex function $\Phi_{Q D N}^{(S)}$. For this we take a simple dipole ansatz, 
$\Phi_{Q D N}^{(S)}\left(x, p_{T}\right) \propto\left(\left(\Lambda_{S}^{2}-m_{Q}^{2}\right) /\left(\Lambda_{S}^{2}-t\right)\right)^{2}$, where $t \equiv-\left(p_{T}^{2}+x\left(m_{D}^{2}-(1-x) M^{2}\right)\right) /$ $(1-x)$. The cut-offs $\Lambda_{S}$ are chosen to reflect the presumably smaller radius of a scalar diquark compared with an $S=1$ diquark: $\Lambda_{0}=1.5 \mathrm{GeV}$ and $\Lambda_{1}=1.2$ $\mathrm{GeV}$. For comparison, we also consider an $s_{Q D}$-dependent dipole vertex function, $\Phi_{Q D N}^{(S)} \propto\left(s_{Q D}+\Lambda_{S}^{2}\right)^{-2}$, and an exponential type, $\Phi_{Q D N}^{(S)} \propto \exp \left(\left(M^{2}-s_{Q D}\right) / \Lambda_{S}^{2}\right)$. These choices are motivated by the resulting symmetry of quark and diquark probability distributions under the interchange $y \leftrightarrow 1-y$ and $m_{Q} \leftrightarrow m_{D}$, which one would need to satisfy if direct scattering from virtual diquarks was included. Since, as mentioned above, these contributions are absorbed into the $Q D N$ vertex function in Fig.1(a), this symmetry need not be explicit, and $t$-dependent vertex functions may be used. The main difference between these forms arises at small $x$, where the $s_{Q D}$-dependent functions give somewhat smaller distributions. The reason for this is the $1 / x$ factor in $s_{Q D}$, which at small $x$ serves to suppress the quark distributions, which themselves depend on inverse powers of $s_{Q D}$. A full investigation of the effects of various vertex functions will be made elsewhere [14.

In Figs.2 and 3 we plot the resulting valence quark distribution in the proton, $x\left(u_{V}+d_{V}\right)=3 x\left(q_{N}^{\operatorname{val}(0)}+q_{N}^{\operatorname{val}(1)}\right) / 2$, as well as the $d_{V} / u_{V}$ ratio. The calculated distribution (dashed curve) has been evolved from $Q_{0}^{2} \approx(0.32 \mathrm{GeV})^{2}$ to $Q^{2}=5$ $\mathrm{GeV}^{2}$ (solid curve) using a contour integration procedure similar to that outlined in Ref.[7] to invert the Mellin transform. The shaded region represents the range of several parametrizations of world data [15]. Clearly the agreement with the data is very good. Even better fits to the data could be obtained by modifying some of the parameters in the analysis, such as diquark masses, form factor cut-offs, or the input scale $Q_{0}^{2}$. Our choices, however, are motivated by physical arguments and previous calculations [6], and we feel the results are encouraging enough to warrant further investigation.

In conclusion, we see that a relativistic constituent quark model formulated in the IMF can lead to reasonable valence quark distributions in the nucleon. Independent of any model approximations, the time-ordered approach used here has the advantage that factorization of subprocesses is automatic. In a covariant formulation the off-shell structure of constituent quarks renders the convolution approach inappropriate. The present work can also be extended to model the sea of the nucleon. Already the diquark-exchange process in Fig.1(c) contributes some 20-30\% 
of the nucleon antiquark distribution at $Q^{2} \sim 5 \mathrm{GeV}^{2}$ [14]. Higher order processes involving multi-pion and multi-diquark exchanges, with correspondingly heavier intermediate states, will further enhance the $x \rightarrow 0$ region. Finally, the spin structure [16] of relativistic constituent quarks can be studied within the same framework; the details of this will appear in a forthcoming publication [14].

W.M. would like to thank H.Meyer, A.W.Schreiber, K.Steininger and A.W.Thomas for discussions related to some of the points addressed in this paper. This work was supported by the BMFT grant 06 OR 735.

\section{References}

[1] R.L.Jaffe, Phys.Rev. D 11 (1975) 1953; R.L.Jaffe and G.G.Ross, Phys.Lett. B 93 (1980) 313; L.S.Celenza and C.M.Shakin, Phys.Rev. C 27 (1983) 1561; C.J.Benesh and G.A.Miller, Phys.Rev. D 36 (1987) 1344; A.W.Schreiber, A.W.Thomas and J.T.Londergan, Phys.Rev. D 42 (1990) 2226; A.W.Schreiber, P.J.Mulders, A.I.Signal and A.W.Thomas, Phys.Rev. D 45 (1992) 3069.

[2] M.Traini, L.Conci and U.Moschella, Nucl.Phys. A 544 (1992) 731; C.J.Benesh, T.Goldman and G.J.Stephenson, Jr., Los Alamos preprint LA-UR-93-1320 (1993); F.M.Steffens and A.W.Thomas, Nucl.Phys. A 568 (1994) 798; H.J.Weber, U.Virginia preprint, hep-ph 9401342.

[3] H.Meyer and P.J.Mulders, Nucl.Phys. A 528 (1991) 589; P.J.Mulders, A.W.Schreiber, and H.Meyer, Nucl.Phys. A 549 (1992) 498; see also A.De Rujula and F.Martin, Phys.Rev. D 22 (1980) 1787, for an early formulation in terms of a non-relativistic vertex function.

[4] S.Weinberg, Phys.Rev. 150 (1966) 1313; see also S.D.Drell, D.J.Levy and T.M.Yan, Phys.Rev. D 1 (1970) 1035.

[5] Y.Nambu and G.Jona-Lasinio, Phys.Rev. Phys.Rev. 122 (1961) 345; T.Hatsuda and T.Kunihiro, Prog.Theor.Phys. 74 (1985) 765; H.Reinhardt and R.Alkofer, Phys.Lett. B 207 (1988) 482.

[6] U.Vogl and W.Weise, Prog.Part.Nucl.Phys. 27 (1991) 195; S.Klimt, M.Lutz, U.Vogl and W.Weise, Nucl.Phys. A 516 (1990) 429. 
[7] M.Glück, E.Reya and A.Vogt, Z.Phys. C 48 (1990) 471; Z.Phys. C 53 (1992) 127; Phys.Lett. B 306 (1993) 391.

[8] V.Bernard, R.L.Jaffe and U.-G.Mei $\beta$ ner, Nucl.Phys. B 308 (1988) 753.

[9] W.Melnitchouk, A.W.Schreiber and A.W.Thomas, Phys.Rev. D 49 (1994) 1183.

[10] N.Ishii, W.Bentz and K.Yazaki, Phys.Lett. B 301 (1993) 165; S.Huang and J.Tjon, Phys.Rev. C 49 (1994) 1702; H.Meyer, Regensburg preprint TPR-94-13.

[11] K.Suzuki, T.Shigetani and H.Toki, TMU-NT931001 (1993), hep-ph/9310266.

[12] F.E.Close and A.W.Thomas, Phys.Lett. 212 B (1988) 227.

[13] S.A.Kulagin, G.Piller and W.Weise, Phys.Rev. C (1994), in print.

[14] W.Melnitchouk and W.Weise, in preparation.

[15] J.G.Morfin and W.K.Tung, Z.Phys. C 52 (1991) 13; J.F.Owens, Phys.Lett. B 266 (1991) 126; A.D.Martin, R.G.Roberts and W.J.Stirling, Phys.Lett. B 306 (1993) 145, Erratum - ibid B 309 (1993) 492.

[16] H.Yabu, M.Takizawa and W.Weise, Z.Phys. A 345 (1993) 193; K.Steininger and W.Weise, Phys.Rev. D 48 (1993) 1433. 


\section{Figure captions.}

1. Deep inelastic scattering from (a) a constituent quark in the nucleon; (b) a constituent quark dressed by pions; (c) a constituent quark dressed by diquarks. The photon four-momentum is $q$, and $P, p$ and $p^{\prime}$ are momenta of the nucleon, constituent quark and spectator diquark, respectively.

2. Calculated valence $x\left(u_{V}+d_{V}\right)$ quark distribution in the proton (dashed), and evolved to $Q^{2}=5 \mathrm{GeV}^{2}$ (solid). The shaded region envelopes the parametrizations of world data from Ref. 15.

3. Ratio of proton $d_{V}$ to $u_{V}$ quark distributions. The curves are as in Fig.2. 
This figure "fig1-1.png" is available in "png" format from: http://arxiv.org/ps/nucl-th/9406037v1 
This figure "fig1-2.png" is available in "png" format from: http://arxiv.org/ps/nucl-th/9406037v1 